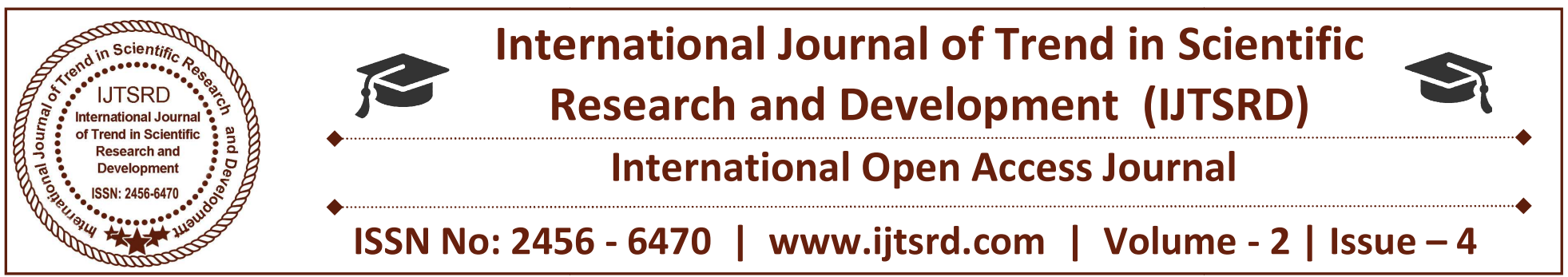

\title{
Medication Adherence of TB Patients using IoT
}

\author{
Manan Abhay Kumar, Sahana S, Soumya K.N \\ Department of Information Science and Engineering \\ School of Engineering and technology, Jain \\ University, Bangalore, India
}

\author{
C. R. Manjunath \\ Dept. of Computer Science and Engineering \\ School of Engineering and Technology- Jain \\ University, Bangalore, India
}

\begin{abstract}
This Project is aimed at proposing a device which does the work of reminding patients with chronic but curable diseases, to take their medicines every day. A large number of people succumb to diseases which are curable due to negligence of adherence to medicines, especially in rural areas of India where the awareness and importance of adherence of medicines is not known. To evade that, the Government of India has come up with a number of plans and schemas. Yet, they fail to give a successful outcome at some point.

Therefore, this paper aims to overcome the cons of the existing system and propose a new idea to make sure that all patients especially the old age ones adhere to the medicine regimen that they have been prescribed. If that happens successfully, it might lead to complete eradication of diseases which are completely curable, and hence are not passed on to the upcoming generations
\end{abstract}

Keywords: DOT; IOT; TB; Tuberculosis; Ministry of Health \& Family Welfare; MoHFW;

\section{INTRODUCTION}

Tuberculosis (TB) is now the leading infectious disease cause of death globally. Multiple studies show that irregular adherence to TB therapy — even for patients who achieve treatment completion or cure is significantly associated with increased rates of disease relapse or development of drug resistance. The strategy of "directly observed treatment" (DOT) was designed to reduce non-adherence and has contributed to substantial improvements in TB treatment outcomes.
The Revised National Tuberculosis Control Program (RNTCP) is the largest tuberculosis (TB) control program in the world based on Directly Observed Treatment Short-Course (DOTS) strategy. Globally, most countries have been using a daily regimen and in India a shift towards a daily regimen for

TB treatment has already begun. The daily strategy is known to improve program coverage along with compliance. fil Such strategic shifts have both management and operational implications.

A major observation that emerged from the survey interviews is that the key to achieve a relapse-free cure is ensuring that a patient receives all doses of the prescribed treatment regimen. However, switching to a daily regimen makes adherence difficult and thus new strategies are needed for its implementation at patient and health provider levels. Most stakeholders appreciate the reasons for switching to a daily regimen. The stakeholders recognized the efforts of the Ministry of Health \& Family Welfare (MoHFW) in spearheading the program. Strategies like the 99 DOTS call-center approach is one of the most effective treatment adherence strategy by far.

\section{A. Problem Statement}

The Government of India is using the 99DOTS scheme, developed by Everwell Health Solutions in Bangalore, India with funding from Bill \& Melinda Gates Foundation, USAID and UKAID. In this system Patients report their adherence to their prescribed medicine regimen using basic mobile handset and toll-free phone calls to phone numbers 
which are revealed only after the medicines are dispensed from the pack. With more than 50,000 patients enrolled primarily in India, 99DOTS is an affordable, scalable and TB-appropriate medication monitoring technology.

\section{The Salient features are:}

Cost-friendly and can be easily integrated into existing drug manufacturer supply chains. 2

When a call from any registered patient number is given, it is marked as dose taken for the day.

The phone numbers are not unique but they just appear in an unpredictable sequence which ensures "pill-in-hand" adherence. This proves to me more accurate than self-reporting via SMS or call.

This way the healthcare providers at the nearest data center can monitor the adherence of the individual patients or a group of patients.

This 99DOTS platform can likewise be incorporated with other adherence screens, empowering suppliers to oversee patients on a solitary stage, free of which observing innovation the patients are utilizing.

The main problem although comes with the way the patients have to report their adherence to their medication. It has been reported to be very tedious for people in rural areas for whom especially this system is directed to. It has been reported that it is difficult for them to give a call every day. And women is rural areas are finding it hard to do this work as well and hence even if they are taking medicines, it is not recorded in the database centers that the patients have adhered to the medication. There are also a number of limitations to this system due to which it has not been able to succeed much in the rural regions.

\section{B. Limitations of the Current Work}

Despite the success of 99DOTS or the Directly Observed Treatment Short-Course, DOT as implemented currently in the resource-limited settings also has limitations:

$>$ Facility-based DOT, still the most common model in many countries, is expensive, resource-intensive and highly burdensome on patients, providers and health systems.
$>$ DOT is often inadequately implemented, with patient self-administration increasingly becoming the norm in both the private and public sector.

$>$ The reminder message sent to the registered mobile number when the patient misses taking the medicine is in English, which the rural people at the grassroots level fail to understand often.

$>$ The dialling and giving a call to the given new number everyday has been recorded to be burdensome by patients.

$>$ Despite the access to a basic phone headset has become very feasible these days, there are still villages where people do not have access to a headset.

Patients especially Women in a few rural backward areas have found using the phone to make a call everyday a hard job.

$>$ If the registered number is not available on a given day and patients send a message from a different number then, the patient's database will not be updated as medicine taken for that specific day when actually the patient would have taken the medicine.

\section{Objectives}

The objective of this project is to propose a new way to record, maintain, and ensure the adherence of patients to their medication by proposing a device which will make the reporting to the data centers about their medicine intake of the day easier than the existing system. By making the system easier, the adherence to the medication and tracking at the data centers, both become easier and productive. This device can be integrated directly with the existing 99DOTS scheme, and can be integrated with any other systems like that as well. This proposal also over comes the limitations of the existing systems and will prove to be more efficient.

\section{LITERATURE SURVEY}

A. The importance of Medication Adherence in improving Chronic-Disease related outcomes: Medication adherence refers to the participation achieved in a medication regimen once an individual has agreed to the regimen. Medication non adherence is chronic conditions is a recognized public health problem. Rates of non-adherence with any medication treatment may vary from $15 \%$ to $93 \%$ with an average estimated rate of $50 \%$. The ramifications of 
medication adherence in affecting patient morbidity are studied and compared across 4 prevalent chronic medical conditions using large patient samples.

B. MediFrame: A Tablet Application to Plan, Inform, Remind and Sustain Older Adults' Medication Intake. Healthcare activities in private home settings that are not supervised by a healthcare professional can challenge older adults. To support such unsupervised care activities, an increasingly number of reminders and monitoring systems are designed. However, most of these systems target a specific treatment or a specific condition. In this paper, we discuss experiences from designing the tablet-based application MediFrame. MediFrame is a personal medication management system to support older adults in non-clinical settings such as the home. The paper describes the user-centered design process and the resulting tablet application. We show how MediFrame can be used to support adherence in medical treatments through fieldwork informed use scenarios. Based on early qualitative feedback, we also discuss lessons learned and how designers can support a holistic medication experience for an older adult's everyday life.

C. A Health IOT Platform based on the integration of Intelligent Packaging, Unobtrusive Bio Sensor, and Intelligent Medicine Box. In-home healthcare services based on the Internet-of-Things (IoT) have great business potential; however, a comprehensive platform is still missing. In this paper, an intelligent home-based platform, the iHome Health-IoT, is proposed and implemented. In particular, the platform involves an open-platform-based intelligent medicine box (iMedBox) with enhanced connectivity and interchangeability for the integration of devices and services; intelligent pharmaceutical packaging (iMedPack) with communication capability enabled by passive radio-frequency identification (RFID) and actuation capability enabled by functional materials; and a flexible and wearable bio-medical sensor device (Bio-Patch) enabled by the state-of-the-art inkjet printing technology and system-on-chip. The proposed platform seamlessly fuses IoT devices (e.g., wearable sensors and intelligent medicine packages) with in-home healthcare services (e.g., telemedicine) for an improved user experience and service efficiency. The feasibility of the implemented iHome Health-IoT platform has been proven in field trials.

D. A Study of Medication-Taking and Unobtrusive, Intelligent Reminding. The objective of this study was to conduct an initial evaluation of a context-aware reminder system, which generated reminders at an opportune time to take the medication. Ten participants aged 65 or older, living alone and managing their own medications, participated in the study. Participants took a low-dose vitamin $\mathrm{C}$ tablet twice daily at times that they specified. Participants were considered adherent if they took the vitamin within 90 minutes (before or after) of the prescribed time. Adherence and activity in the home was measured using a system of sensors, including an instrumented pillbox. There were three phases of the study: baseline, in which there was no prompting; time-based, in which there was prompting at the prescribed times for pill-taking; and context-aware, in which participants were only prompted if they forgot

to take their pills and were likely able to take their pills. The context-based prompting resulted in significantly better adherence $(92.3 \%)$ as compared to time-based $(73.5 \%)$ or no prompting (68.1\%) conditions $\left(p<0.0002, \chi^{2}=17.0\right)$. In addition, subjects had better adherence in the morning than in the evening. We have shown in this study that a system that generates reminders at an opportune time to take the medication significantly improves adherence.

\section{DESIGN METHODOLOGY}

The design of the proposed method is such that it will make the existing tedious work of dialing a different phone number every day and give a call till you get an acknowledgment so that the health care department make a note of it that, that particular patient has taken the medicine for the day, easy.

The design of the proposed system includes a device which should be given by the health care centers with a sim card whose numbers are recorded and inserted into database with the details of the patient it is been given to.

The sim card will be inserted into the device. The device will have an in-built alarm system which will alert twice a day for which it will have a stop button. Along with that button it will have a message button which when pressed a message should be sent to the heath care centers confirming that they have taken the medicine for the day. The device will be based on IOT and hence will be programmed to send a message on the press of the button. And the message will have to be sent twice a day after every dose of the medicine. This way the patients do not have to 
remember to take their pills and instead the device will remind by setting the alarm off and making the patient stop it and take the pill and send the message.

This design makes it easy for the illiterate also in the rural regions of India to use. They will not have to dial any numbers and make a phone call from a mobile handset and not worry about making the call from the registered phone number.

\section{THE PROPOSED SYSTEM}

The aim of the new proposes remain the same of following a daily regimen to ensure adherence to the medication by all patients, but in an easier way by the use of an IOT device.

\section{A. What is IoT?}

The Internet of Things (IoT) is the network of physical devices, vehicles, home appliances and other items embedded with electronics, software, sensors, actuators, and connectivity, which enables these objects to connect and exchange data. Each thing is uniquely identifiable through its embedded computing system and is able to inter-operate within existing internet infrastructure.

\section{B. Why is medicine adherence important?}

When a patient adheres to their medicine regimen, they benefit not only themselves and their family, but also their county and government. The healthier citizens of a country are, the better the economy proves to be. When a patient adheres to their medicine regimen, they can decrease their dosage, frequency, follow-up check-ups and most importantly better their health.

This problem is not only in India but most of the countries in the world face this. The main problem being the people who are prescribed the medications do not understand the importance of adherence to it. According to New York Times 20 percent to 30 percent of medication prescriptions are never filled, and that almost 50 percent of medications for chronic disease are not taken as prescribed. Even people who take prescription medications be it for a simple infection, cold, or a life threatening condition, typically take only about half the prescribed dosage.

This lack of adherence is known to cause a large number of deaths and hospitalizations which affects people economically, mentally and physically.

\section{Block Diagram of the proposed system:}

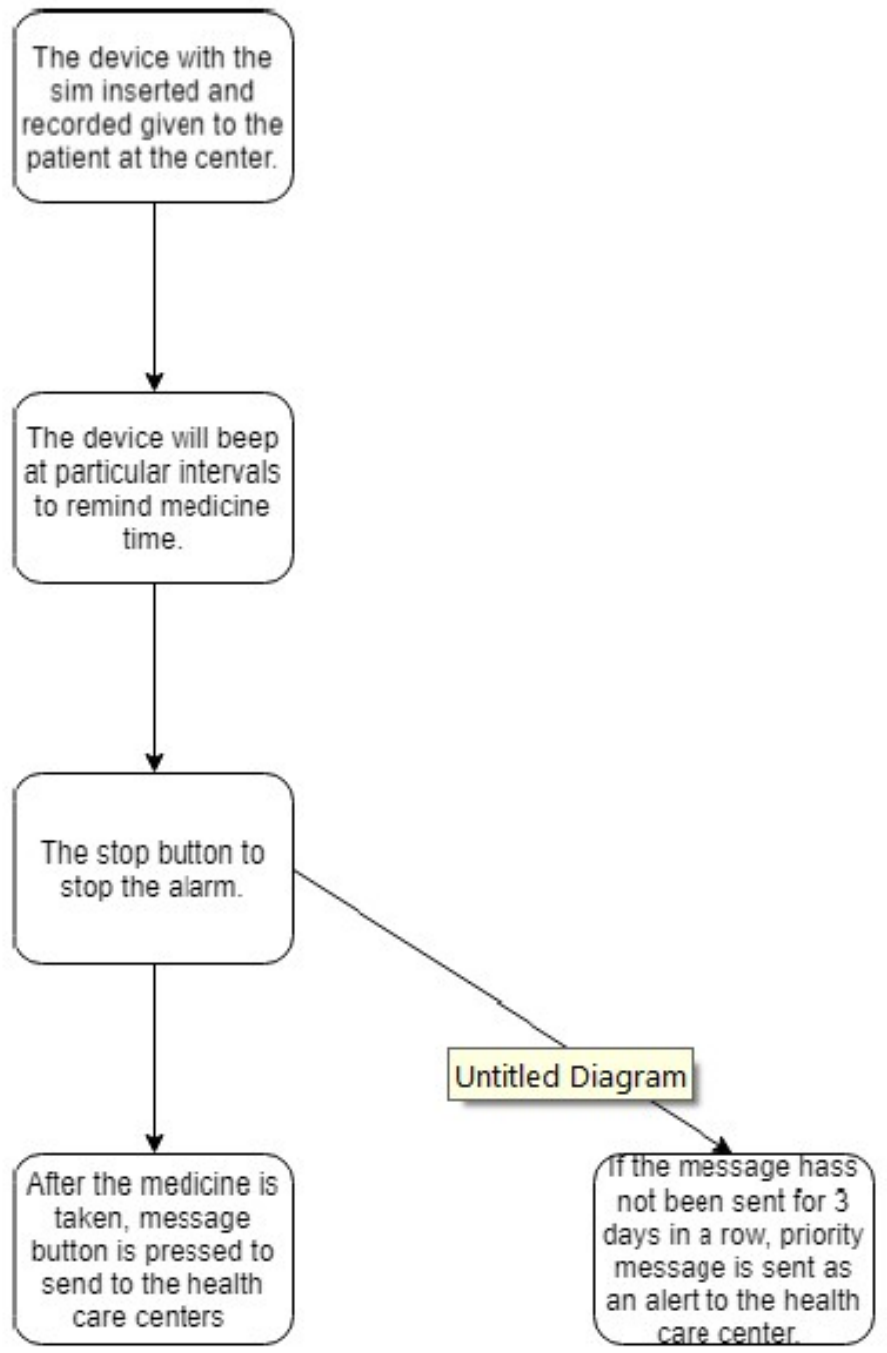

Fig 4.3: Block Diagram of the proposed system

\section{Features of the proposed system:}

$>$ An embedded device which will hold a SIM card will be given by the centre where the number will be stored in the database of the centre.

The number of patients taking the medication in that particular house will also be noted and registered under that number.

$>$ At the time of giving the device, the usual timings of taking the medicine is asked and set on the device, and at that particular time the device will beep reminding the patients to take the medicine.

$>$ After taking the medicine depending on the number of the people under medication, the button has to pressed that many times, and a message from the number will be sent to 
the centre confirming that the medicine has been taken.

$>$ So according to the dosage (which is twice a day) the reminder will be sent twice a day at the expected time of medicine intake. The reminder will be like an alarm which will have to be stopped by pressing the stop button.

$>$ Another button will for sending the message after having taken the medicine which is to be done only after the dose of the day has been taken, i.e twice a day.

$>$ The message sent by the device will have time and a standard message which will confirm to the centre that the pill has been taken.

The patients will be able to send the message only after the 2 alarms i.e after the two pills of the day.

And when the pill hasn't been taken for more than 3 times in a week, a priority alert goes to the centre informing that the medicine has been missed more than 3 times in a week, and actions will be taken accordingly.

$>$ The message sending system becomes hassle free by using only a button to intimate the data centres that the medicine at that particular house $\mathrm{h}$ ass been taken.

$>$ There is no confusion that which number does the call has to be done from. The device will come with a sim whose number is recorded in the centre before been given to the patients.

$>$ The device will remind the patient to take medicine by setting an alarm which is set to the average expected time the patient takes their medicine depending on their dosage.

This system makes it easier for everyone to use, old or young, man or woman, in rural or urban areas.

$>$ This system will make sure the adherence of patients on all dosages as opposed to the existing system where they have to give a call only after the last dosage of the day.

$>$ The same device can be used to record for the number of patients in one house taking medications. The buttons can be pressed the number of times the people are taking one dosage at a time.

\section{E. Working of the proposed system}

The system proposed in this project is based on Internet of Things or IoT. This system focuses on how to integrate technology of the Internet of Things with the existing systems following some out of date technology. This will help not only help the users but also the one who maintain and monitor such systems.

In the existing system referenced in this paper, there are health care centres in rural areas where there has been reported high rates of TB. These health care systems have a social worker called "ASHAWORKER" whose job is to go around the village and survey who has the disease, who has symptoms of the disease, who needs attention with the symptoms they are showing, who is aware of the disease, who is aware of the importance of the adherence of medication for the disease and who are adhering to their medication regimen, and then submit this report to the health care centre. Then the patients who are under medication. The one who are showing symptoms are called to the health care centres and given an awareness lesson about how the disease is completely curable but yet people over generation and generation have been suffering and even facing deaths by it. They are told how important adhering to their regimen is, as it can completely eradicate the disease and no one in the future generation in their family or any family in the country will have.

After this, all details of the patient are collected in a database like their age, family history of the disease, duration of the medication before this, etc. Then the device which is proposed should be given to the patient and the number of the SIM card inserted in it noted down along with the number of patients in the house the device is going to. The working of the device is explained to the patient and the care-taker or any other member of the family as well.

The device will beep to remind the patient to take medicines when it is the time to as set the health care worker, and the patient has to stop the alarm, supposed to take the medicine and then press the second button on it in order to send a message to the data centre confirming that one dose of the day has been taken. Similarly, the routine is repeated second time of the day at night, when the device's alarm will be set off which the patient has to stop and take the 
medicine and press the second button acknowledging that the second dosage of the day has been taken.

Hence, this way the adherence of the patients to their prescribed medication regimen can be ensured. TB has the same dosage and tablets more or less on any situation and criteria which makes it easy for any health care centre to manage the device and set the alarm on an average time of their intake.

Many factors may influence patients' adherence to the medication intervention Patients' demographics, cost of medications, number of medications taken by the patient, nature of medical condition, and the nature of the medical intervention. Patients failing to adhere to the medicine regimen may cost as much as $\$ 300$ billion to the healthcare system of a country.

\section{CONCLUSION AND FUTURE SCOPE}

The conclusion of this project is that this system proposed in this paper can make the adherence system of the TB-HIV positive patients to their medication much easier than the existing system as run by the Government Of India. This system makes the entire routine of letting the health care workers know that the daily dosage of the medicine has been taken, easier.

The proposed device can not only be used for this system, but also can be integrated with any such future and many more existing systems initiated by the Government like the Mother and Child care Tracking system, etc. These schemes for eradication is mainly directed at the patients in the rural areas as the awareness of the disease and medication and adherence to the medication is very low at these rural areas and hence there is still a high mortality rates due to such diseases which are otherwise completely curable.

The future scopes of this proposal is very wide as this system can be implemented and integrated into the existing system very easily and be used for any such kinds of schemes proposed in the future.

\section{FUTURE SCOPE}

In future this system can be improvised to make the messages being sent to the centers to a single message no matter how many ever patients are there in that particular mouse. A counter button can be added which can be set to how many medicines have been consumed for that dosage time and sent one collective message to the center.

Or the system can be made to send a message only once a day after the second dosage has been taken. Although this might seem like patients will play with the loopholes in this way but it will go easier on the data centers of the health care centers.

Or the system can be improvised to reveal a single digit number when every pill of medicine is dispensed from the package and that number will have to be set on the device using the counter button and then send to the center. This number sent in the message can be matched in the data center. This numbers in the database can be saved as a pattern which will be associated with the strip of the medicine. A barcode can be generated on each strip of medicine which can be scanned in the health centers when it is been given to the patients and the unique number of the strip and the not compulsorily unique pattern is recorded in the database with the sim card number of the patient so that when the count is being received by the patients when using, it can be matched and ensured that patients are not bluffing the message sending. The pattern must be random and different but need not be unique.

\section{ACKNOWLEDGEMENT}

It is a great pleasure for us to acknowledge the assistance and support of a large number of individuals who have been responsible for the successful completion of this seminar work.

First, we take this opportunity to express our sincere gratitude to School of Engineering \& Technology, Jain University for providing us with a great opportunity to pursue our Bachelor's Degree in this institution.

In particular we would like to thank Dr. Hariprasad $\mathbf{S}$ A, Director, School of Engineering \& Technology, Jain University for his constant encouragement and expert advice.

We express our sincere regards and gratitude to Dr.Rajasimha A Makaram, Associate Director, School of Engineering \& Technology, Jain University for his timely help and inspiration throughout this work.

It is a matter of immense pleasure to express our sincere thanks to Dr. Santosh Naik, Head of the 
department, Computer Science and Engineering, Jain University, for providing right academic guidance that made our task possible.

We would like to thank our Seminar coordinators Mr.Manjunath $C \quad R$, Associate Professor, Department of Computer Science and Engineering, Mrs. Soumya K N, Assistant Professor, Department of Information Science and Engineering and Mrs. Sahana Shetty, Assistant Professor, Dept. of Computer Science and Engineering, Jain University, for sparing his/her valuable time to extend help in every step of our seminar work.

We would like to thank all the staff members of Computer Science and Engineering and Information Science and Engineering for their support.

We also extend our sincere gratitude to all the Library Staff for extending their help and support towards the preparation of the plagiarism report for our work.

\section{REFERENCES}

1. Phillip A. Laplante, Mohamad Kassab, Nancy L. Laplante, Jeffrey M. Voas, " Building Caring Healthcare Systems in the Internet of Things", IEEE Systems Journal, 22-February-2017, Page: $1-8$

2. Kunal Dhariwal, Ashish Mehta, "Architecture and Plan of Smart Patient Monitor based on IOT" , IRJET, Vol 04, Issue 04, Apr-2017, Page: 23950056

3. Latif, A.I., Othman, M., Ali, N.B., Suliman, A. ,Mahdi, O.A., "An investigation / of IOT importance in maintaining health records and retrieval using electronic tags", CSIT Scholarly Publication, 2016 , http://dspace.uniten.edu.my/jspui/handle/1234567 $89 / 8744$

4. Jisha S, Mintu Philip, " RFID based security platform for Internet Of Things in health environment for patients", IEEE, 4 May 2017, Coimbatore, India.

5. SumanBhattacharyaa, DavidWainwrighta, JasonWhalleya, “ Internet of Things enabled assistive care services" , ELSEVIER, August 2017.

6. A Murali, K Raj, A Asifa, AS Nair, C Vincent, “ The smart Health Medicare System to track patient medical and health needs", IJSCE, Vol-5, Issue-4, Page 75-77, April 2017

7. S. Khicha, P. Patil, P. Roy, P. Sinkar, J P Rankhambe, "A Mobile Based Smart Healthcare System", International Journal Of Engineering And Computer Science, Vol. 6, Issue. 4, pp. 20911-20914, April 2017.

8. G. Alex, B. Varghese, J.G. Jose, A. Abraham, “A Modern Health Care System Using IoT and Android", International Journal on Computer Science and Engineering (IJCSE), Vol.8, Issue.4 pp. 117-121, Apr 2016.

9. Ruiling Gao, Minghuan Zhao , Zhihui Qiu , Yingzhou Yu and C. Hwa Chang, " An Improved Motion-Sensing based monitoring system for patients using IOT" , International Journal of Software Innovation, 2015.

10. R.M. Liaqat, A. Athar, N.A. Saqib "Intelligent Agent based System for Monitoring of medicine regimen of patients", 2015 2nd International Conference on Information Science and Security (ICISS), South Korea, pp. 978- 982, 2015. 